\title{
Radiation Dose Reduction During Adrenal Vein Sampling Using a New Angiographic Noise Reduction Imaging Technology
}

\section{Clemens Spink ( $\nabla$ c.spink@uke.de )}

University Medical Center Hamburg-Eppendorf

\section{Maxim Avanesov}

University Medical Center Hamburg-Eppendorf

\section{Alexander Lenz}

University Medical Center Hamburg-Eppendorf

\section{Frank Oliver Henes}

University Medical Center Hamburg-Eppendorf

\section{Lennart Well}

University Medical Center Hamburg-Eppendorf

\section{Thomas Schmidt}

Philips (Germany)

\section{Michael Grass}

Philips (Germany)

\section{Gerhard Adam}

University Medical Center Hamburg-Eppendorf

\section{Harald Ittrich}

University Medical Center Hamburg-Eppendorf

\section{Peter Bannas}

University Medical Center Hamburg-Eppendorf

\section{Research Article}

Keywords: Adrenal vein sampling, radiation dose, image-processing technology, interventional radiology, angiography

Posted Date: May 7th, 2021

DOl: https://doi.org/10.21203/rs.3.rs-446113/v1

License: (c) (1) This work is licensed under a Creative Commons Attribution 4.0 International License.

Read Full License 


\section{Abstract}

Purpose: To compare the patient radiation doses during angiographic selective adrenal vein sampling (AVS) before and after a noise reduction imaging technology upgrade.

Methods: In this retrospective single-center-study, cumulative air kerma (AK), cumulative dose area product (DAP), fluoroscopy time and contrast agent dosage were recorded from 70 patients during AVS. 35 procedures were performed before and 35 after a noise reduction imaging processing technology upgrade. Mean values were calculated and compared using an unpaired student's t-test. DSA image quality was assessed independently by two blinded readers using a four-point Likert scale (1=poor; 4=excellent) and compared using Wilcoxon signed-rank test.

Results: After the technology upgrade we observed a significant reduction of $35 \%$ in AK $(1.7 \pm 0.7 \mathrm{vs}$. $1.1 \pm 0.7 \mathrm{~Gy}, \mathrm{p}=0.01)$ and a significant reduction of $28 \%$ in DAP $\left(235.1 \pm 113\right.$ vs. $\left.170.1 \pm 94 \mathrm{~Gy}^{*} \mathrm{~cm}^{2}, \mathrm{p}=0.01\right)$ in comparison to procedures before the upgrade. There were no significant differences between the number of exposure frames ( $143 \pm 86$ vs. $132 \pm 61$ frames, $p=0.53)$, fluoroscopy time ( $42 \pm 23$ vs. $36 \pm 18$ min, $p=0.22)$, or the amount of contrast medium used $(179.5 \pm 84$ vs. $198.1 \pm 109 \mathrm{ml}, \mathrm{p}=0.41)$. There was also no significant difference regarding image quality (3 (2-4) vs. $3(2-4), p=0.67)$.

Conclusion: The angiographic noise reduction imaging technology upgrade significantly decreases the radiation dose during adrenal vein sampling without increasing time of fluoroscopy or contrast volume and without compromising image quality.

\section{Key Points}

- Noise reduction technology reduces radiation exposure by one third in adrenal vein sampling.

- Noise reduction technology does not reduce image quality.

- Noise reduction technology does not increase procedure duration or amount of contrast agent used.

\section{Introduction}

Primary aldosteronism (PA) is the most frequent secondary form of hypertension and is characterized by an autonomous adrenocortical over secretion of aldosterone (1). PA has a prevalence of up to $11 \%$ in hypertensive patient populations $(2,3)$. The aldosterone excess either results from bilateral idiopathic adrenal hyperplasia (IHA) or unilateral aldosterone-producing adenoma (APA) (4).

The appreciation that primary aldosteronism is far more common than previously recognized and the essential distinction between APA and IHA results in an increasing requirement for adrenal vein sampling (5).

Angiographic selective adrenal vein sampling (AVS) allows identification of patients with primary aldosteronism by direct sampling of hormones produced by each gland (8). Patients with APA can benefit 
from adrenalectomy, while IHA-patients require conservative drug treatment (4). Removing the identified gland producing excess adrenal hormone may cure secondary hypertension, thereby significantly improving patient's prognosis regarding cardiovascular complications and renal function (9).

Unfortunately, diagnostic angiographic procedure time is relatively long because AVS is a technically demanding procedure: AVS may be prolonged because of anatomic variations of adrenal vein ostia, particularly of the right adrenal vein (10). A prolonged search for venous ostia can result in long fluoroscopy times up to 184 minutes with high dose area products (DAP) up to $3181 \mathrm{~Gy}^{\star} \mathrm{cm}^{2}(11)$.

Different techniques of radiation dose reduction for angiographic procedures have been developed over the past years (12-15). Particularly the potential of noise reduction by image-processing have been successfully introduced and recently advanced further (16). Real-time post-processing imaging algorithms allow reduced radiation doses, while maintaining adequate image quality (17). However, the degree of dose reduction varies and depends on the examined body region. Specific post-processing imaging algorithms offer tailor-made settings depending on the particular field of intervention, such as noise reduction, motion compensation and improved edge imaging. Motion compensation is extremely useful during thoracic or upper abdominal interventions in order to reduce breathing artifacts. Algorithms for stable interventions such as cerebral or peripheral angiography mainly benefit from noise reduction and improved edge imaging. Published dose reductions range from $43 \%$ during cardiac interventions up to $83 \%$ during iliac angiography (16-18). Furthermore, significantly reduced radiation doses up to $59 \%$ during bronchial artery embolization as well as up to $57 \%$ during intrahepatic interventions such as transjugular intrahepatic portosystemic shunts have been reported $(19,20,21)$.

Up to now, the dose reduction potential of the AlluraClarity upgrade has not been assessed in patients undergoing AVS. The aim of our study was therefore to compare radiation doses in patients undergoing AVS between AlluraClarity and the precursor technology.

\section{Materials And Methods}

\section{Study design and patient selection}

Our retrospective single-center-study was approved by the local institutional review board "Aerztekammer Hamburg". Informed consent of all study participants had been obtained. All procedures were performed in accordance with the standards of the act for healing professions of Hamburg, Germany with the principles of the 1964 Declaration of Helsinki and its following amendments. All clinical imaging data were anonymized. The anonymization of patient data in the research process ensured data protection in accordance with the European General Dara Protection Regulation.

Between June 2012 and August 2018, a total of 91 AVS procedures have been performed at our department on patients with suspected primary aldosteronism using an Allura FD20 angiographic system (Philips Healthcare, Best, Netherlands). 
In June 2014 the angiographic image-processing technology received an upgrade from "Allura Xper" to "AlluraClarity" (Philips Healthcare, Best, The Netherlands).

Our study group was recruited from 44 patients undergoing AVS between June 2014 and August 2018 after the AlluraClarity upgrade. Nine of these 44 patients $(20.5 \%)$ had to be excluded due to incomplete unilateral left adrenal vein sampling. The remaining 35 patients with successful sampling of both left and right AVS during a single AVS procedure resulted in our study group.

Our control group was recruited from 47 patients undergoing AVS between June 2012 and May 2013 before the AlluraClarity upgrade. Twelve of these 47 patients $(25.5 \%)$ had to be excluded due to incomplete unilateral left adrenal vein sampling. The remaining 35 patients with successful sampling of both left and right adrenal veins during a single AVS procedure resulted in our control group (Table 1).

Table 1

Patient demographics.

\begin{tabular}{|llll|}
\hline Patient characteristics & Allura Xper & AlluraClarity & p-value \\
\hline Number of patients & 35 & 35 & \\
\hline Female & $15(42 \%)$ & $16(45 \%)$ & \\
\hline Age $(\mathrm{y})$ & $58.1 \pm 13.2$ & $54.4 \pm 9.5$ & 0.13 \\
\hline Body mass index $\left(\mathrm{kg} / \mathrm{m}^{2}\right)$ & $27.1 \pm 5.2$ & $28.8 \pm 3.9$ & 0.35 \\
\hline
\end{tabular}

Note - Data presented as mean \pm standard deviation; * = significant. None of the included patients underwent AVS twice.

\section{Adrenal vein sampling (AVS)}

AVS is a standardized procedure at our institution in accordance to the Endocrine Society guidelines and expert consensus statement of the American Heart Association $(6,7)$. Aldosterone release was stimulated before and during AVS with synacthen, a synthetic signal peptide of adrenocorticotropic hormone (ACTH) in a $50 \mu \mathrm{g} / \mathrm{h}$ infusion. The ACTH infusion prior the AVS provides the advantage of constant adrenal stimulation which levels circadian deviation (6).

Sampling was performed in all cases by board certified radiologists with 8-20 years of experience in interventional radiology. Details of the procedure are described in detail elsewhere (5). First, blood samples $(5 \mathrm{ml})$ from the inferior vena cava (IVC) were collected as a reference. Second, the left adrenal vein ostium was carefully probed selectively with 5F-Cobra or 5F-Aachen-I catheters (Radifocus, Terumo, Japan). Third, the right adrenal vein ostium was carefully probed selectively with a 5F-Mikaelsson catheter (Impress, Merit Medical, USA) or 5F/4F-Sidewinder or 5F-Cobra catheters (Radifocus, Terumo, Japan). Venograms were performed on both left and right adrenal veins using 3-5 $\mathrm{ml}$ contrast agent (Imeron 300, Bracco, Italy) to document successful probing of adrenal vein ostia. Blood samples (5 ml) were collected from each adrenal vein ostium. Intraprocedural cortisol measurements confirmed 
successful adrenal vein sampling. Finally, IVC blood samples $(5 \mathrm{ml})$ were collected as a second and final reference.

\section{Imaging systems}

The imaging technology AlluraClarity upgrade improves noise reduction by both optimized hardware and real-time image processing-algorithms, tailor-made for different body areas by adjusted acquisition parameters (16). Compared to the precursor technology hardware changes include additional filtering of $1.0 \mathrm{~mm}$ aluminum und 0.1 copper and a reduced tube current for fluoroscopy and digital subtraction angiography (DSA). Further, during fluoroscopy the tube voltage is decreased from 80 to $70 \mathrm{kV}$. Different fluoroscopy settings ranged from low to medium to high dose include increasing frames per second (fps) and decreasing levels of filtration (Table 2). After the technology upgrade, the pulse width was halved from 7.0 to $3.5 \mathrm{~ms}$ resulting in shortened pulses during fluoroscopy. The focal spot size was decreased from 7.0 to $3.5 \mathrm{~mm}$ during DSA to improve spatial resolution. 
Table 2

Imaging settings for abdominal fluoroscopy and DSA.

\begin{tabular}{|c|c|c|}
\hline Fluoroscopy & Allura Xper & AlluraClarity \\
\hline Total filtration & $3 \mathrm{~mm} \mathrm{Al}+0.4 \mathrm{~mm} \mathrm{Cu}$ & $4 \mathrm{~mm} \mathrm{Al}+0.5 \mathrm{~mm} \mathrm{Cu}$ \\
\hline Pulse width (ms) & 7 & 3.5 \\
\hline Tube current (mA) & 160 & 60 \\
\hline Tube voltage $(\mathrm{kV})$ & 80 & 70 \\
\hline \multicolumn{3}{|l|}{ Fluoroscopy modes } \\
\hline \multicolumn{3}{|c|}{ Frames per second (fps) and filtration } \\
\hline Low dose & $3 \mathrm{fps}$ & $3 \mathrm{fps}$ \\
\hline (default) & $3 \mathrm{~mm} \mathrm{Al}+0.4 \mathrm{~mm} \mathrm{Cu}$ & $4 \mathrm{~mm} \mathrm{Al}+0.5 \mathrm{~mm} \mathrm{Cu}$ \\
\hline \multirow[t]{2}{*}{ Medium dose } & $7 \mathrm{fps}$ & $7 \mathrm{fps}$ \\
\hline & $3 \mathrm{~mm} \mathrm{Al}+0.4 \mathrm{~mm} \mathrm{Cu}$ & $4 \mathrm{~mm} \mathrm{Al}+0.4 \mathrm{~mm} \mathrm{Cu}$ \\
\hline \multirow[t]{2}{*}{ High dose } & $15 \mathrm{fps}$ & $15 \mathrm{fps}$ \\
\hline & $3 \mathrm{~mm} \mathrm{Al}+0.1 \mathrm{~mm} \mathrm{Cu}$ & $4 \mathrm{~mm} \mathrm{Al}+0.1 \mathrm{~mm} \mathrm{Cu}$ \\
\hline DSA & Allura Xper & AlluraClarity \\
\hline Total filtration & $3 \mathrm{~mm} \mathrm{Al}+0.4 \mathrm{~mm} \mathrm{Cu}$ & $4 \mathrm{~mm} \mathrm{Al}+0.5 \mathrm{~mm} \mathrm{Cu}$ \\
\hline Focal spot (mm) & 7 & 3.5 \\
\hline Tube current (mA) & 160 & 60 \\
\hline Tube voltage $(\mathrm{kV})$ & 80 & 80 \\
\hline Frames per second (fps) & 2 & 2 \\
\hline
\end{tabular}

Apart from hardware changes, the new technology uses different background post-processing algorithms during image acquisition. These algorithms include temporal and spatial noise reduction techniques as well as motion compensation and improved edge imaging (17).

\section{Radiation dose measurement and documentation}

The dose area product (DAP) defines the amount of dose absorption of an irradiated area in $\mathrm{Gy}^{\star} \mathrm{cm}^{2}$, measured by ionization chambers placed nearby X-ray collimators (23). The DAP is independent of the distance from the radiation source.

Kerma, the kinetic energy released in matter can be calculated in the air at the interventional reference point (IRP) in units of Gray (Gy) depending on the DAP, the collimation of a field, the tube voltage, the tube current, and the source to image-receptor distance. The IRP is located along the X-ray beam at a distance 
of $15 \mathrm{~cm}$ above the isocenter in direction from the X-ray tube towards the detector. Therefore, the air kerma (AK) can be used as a surrogate for the patient's skin dose (22).

During each interventional procedure different parameters including AK, DAP and fluoroscopy time were collected automatically within Radiation Dose Structured Reports (RDSR). After each intervention these reports were transferred into the Picture Archiving and Communication System (PACS) (24). Additionally the amount of contrast agent was withdrawn from the documented list of material usage.

\section{Image quality assessment}

Qualitative DSA image analysis was performed by two radiologists with 3 years of experience in interventional radiology each. Both readers were blinded to the imaging technology and assessed randomized venograms of adrenal veins. Both readers used the same working station monitor with maintained window and image settings at default alignments. The two readers determined separately whether each venogram satisfied the following findings: sharp delineation of the adrenal veins with sufficient contrast, contrast stain of the adrenal gland, low level of subjective image noise. The readers scored the images using a four-point Likert scale adapted from Morita et al. 2017 (13): 4 = excellent, all findings were observed; 3 = good, two of the findings were observed; 2 = fair, one of the findings was observed; 1 = poor, no findings were observed.

\section{Statistical analysis}

Mean or median values of recorded values as well as standard deviations (SD) or ranges were calculated as appropriate. Results of AK, DAP, fluoroscopy time, age and BMI were compared using an unpaired student's t-test. Results of Likert scale variables were compared using a Wilcoxon-Mann-Whitney test. Cohen's weighted kappa was calculated for inter-rater reliability. P-values $<0.05$ were considered statistically significant. Statistical analyses were performed with Quick Calcs 2015 (Graphpad Software, La Jolla, CA) and SPSS 22.0 (IBM Corp., Armonk, NY).

\section{Results}

\section{AVS procedures}

There was no significant difference regarding age ( $54.4 \pm 9.5$ vs. $58.1 \pm 13.2 \mathrm{y}, \mathrm{p}=0.13)$ or BMI $(28.8 \pm 3.9$ vs. $27.1 \pm 5.2 \mathrm{~kg} / \mathrm{m}^{2}, p=0.35$ ) between the study group (AlluraClarity) and the control group (Allura Xper) (Table 1). There was no significant difference regarding time of fluoroscopy (36 \pm 18 vs. $42 \pm 23 \mathrm{~min}, p=$ 0.22 ) or the amount of contrast medium used (198 $\pm 109 \mathrm{vs.} 179 \pm 84 \mathrm{ml}, \mathrm{p}=0.41)$ between the study group (AlluraClarity) and the control group (Allura Xper) (Table 3 ). 
Table 3

Procedure parameters during AVS.

\begin{tabular}{|lllll|}
\hline Parameters & Allura Xper & AlluraClarity & Percentage difference & p-value \\
\hline Cumulative DAP $\left(\mathrm{Gycm}^{2}\right)$ & $235.1 \pm 113.1$ & $170.1 \pm 94.5$ & $-28 \%$ & $0.01^{\star}$ \\
\hline Cumulative AK (Gy) & $1.7 \pm 0.7$ & $1.1 \pm 0.7$ & $-35 \%$ & 0.01 * \\
\hline Number of exposure frames & $143 \pm 86$ & $132 \pm 61$ & $-8 \%$ & 0.53 \\
\hline Fluoroscopy time (min) & $42.3 \pm 23.1$ & $36.5 \pm 18.6$ & $-14 \%$ & 0.22 \\
\hline Contrast agent volume $(\mathrm{ml})$ & $179.5 \pm 84.0$ & $198.1 \pm 109.1$ & $+10 \%$ & 0.41 \\
\hline
\end{tabular}

Note - Data presented as mean \pm standard deviation, * = significant.

\section{Radiation dose}

After the technology upgrade from Allura Xper to AlluraClarity the mean DAP was reduced by $28 \%(235.1$ \pm 113.1 vs. $170.1 \pm 94.5 \mathrm{~Gy}^{*} \mathrm{~cm}^{2}, p=0.01$ ) (Fig. 1). The mean AK also decreased by $35 \%$ (1.7 $0.7 \mathrm{vs.} 1.1$ $\pm 0.7 \mathrm{~Gy}, \mathrm{p}=0.01$ ) after the technology upgrade from Allura Xper to AlluraClarity (Table 3 ).

\section{DSA Image quality}

Comparing Allura Xper and AlluraClarity no significant difference regarding DSA image quality could be observed with a median 3 vs. 3 (Interquartile range (IQR) $2-4$ vs. $2-4, p=0.67$ ) in Fig. 2. Image quality ratings demonstrated a comparable level of inter-rater agreement for image quality ratings of AlluraClarity $(\mathrm{k}=0.77)$ and Allura Xper $(\mathrm{k}=0.75)$ (Table 4).

Table 4

DSA image quality of adrenal venograms.

\begin{tabular}{|lllll|}
\hline Imaging technology & $\begin{array}{l}\text { Average } \\
(\mathrm{IQR})\end{array}$ & $\begin{array}{l}\text { Reader } 1 \\
(\mathrm{IQR})\end{array}$ & $\begin{array}{l}\text { Reader } 2 \\
(\mathrm{IQR})\end{array}$ & Weighted Kappa (95\%-Cl) \\
\hline Allura Xper & $3(2-4)$ & $3(2-4)$ & $3(2-4)$ & $0.75(0.60-0.89)$ \\
\hline AlluraClarity & $3(2-4)$ & $3(2-4)$ & $3(2-4)$ & $0.77(0.60-0.94)$ \\
\hline $\begin{array}{l}\text { p-value } \\
\text { Allura Xper vs. AlluraClarity }\end{array}$ & 0.67 & 0.94 & 0.52 & \\
\hline
\end{tabular}

Note - Data presented as mean with interquartile range (IQR) or with $95 \%$ confidence intervals (CI); * significant. Image quality was graded as follows: 1 , poor; 4 , excellent.

\section{Discussion}


Our study demonstrates that the AlluraClarity technology upgrade enables a radiation dose reduction up to one third in patients during AVS compared to the precursor technology Allura Xper without compromising image quality.

It is conceivable that the main reason for a significant reduction of radiation dose after the upgrade using AlluraClarity results from the additional filters of aluminum and copper during DSA and a reduced focal spot size during fluoroscopy. By changing system parameters AlluraClarity renders over 500 different acquisition settings with respect to the particular area of interest. Various studies have already demonstrated the potential in radiation dose reduction using AlluraClarity during intracranial angiography, cardiac angiography, iliac angiography or abdominal angiography (16-19). Apparently, procedural settings during cerebral interventions focus more on the pixel shift feature during steady conditions, as thoracic and especially abdominal settings emphasis more on the motion control feature in order to reduce breathing artifacts (20).

Previous studies of abdominal interventions such as transarterial chemoembolization (TACE) and transjugular intrahepatic portosystemic shunt (TIPS) using AlluraClarity abdominal settings reported dose reduction of $>50 \%(19,21)$. We observed a lower dose reduction of only $28 \%$ using the identical AlluraClarity abdominal settings during AVS.

The most important reason for less dose reduction during AVS might be due to its different anatomic region and the complexity of intervention. The most challenging part of AVS remains the reliable cannulation of the right adrenal vein (RAV) (10). Identifying the RAV-ostium from the IVC with 1-2 mm diameters requires increased contrast of the vessel edges and therefore increased radiation dose because of increased fluoroscopy time during the search of the RAV-ostium.

Furthermore, AlluraClarity provides three different levels of fluoroscopy-setups at the operator's console ranging from low and medium to high dose settings. These changes can be applied differently for each fluoroscopy-run and might change due to individual preferences from operator to operator (Table 2). Unfortunately, these specific individual sub-settings are currently not monitored by the vendors dose reports and could not be tracked retrospectively. The default settings contain the low dose profile, which can be increased individually.

The relative increase in contrast agent used by $10 \%$ for additional DSA- or fluoroscopy runs may be caused by the verification of correct catheter placement before and after sampling (Table 3). Our results show a significant radiation dose saving potential after the upgrade to AlluraClarity with a mean DAP decreasing from 235.1 to $170.1 \mathrm{~Gy}^{*} \mathrm{~cm}^{2}$. Recently the SPARTACUS multicenter trial released comparable mean AVS procedure doses from the identical fluoroscopy system AlluraClarity of 147 (1.1-1186) $\mathrm{Gy}{ }^{*} \mathrm{~cm}^{2}(11)$.

The main limitation of our study is the variety of different operators involved over 4 years due to its retrospective nature. Although AVS is to be considered as a highly standardized procedure, operators at different skill levels use their personal workflow of intervention during AVS. Evidently, identical and 
matched operators should have performed the interventions for both groups of patients before and after the technology upgrade. For this study we used our standard AVS-protocol. Busser et al. could already demonstrate potential dose reduction and reduced DSA-runs via intraprocedural CBCT image registration guidance improving the detection of adrenal vein ostia (14). However, this new feature of image registration is currently not practiced at our angiography suite and therefore has not been included in our routine AVS-protocol yet. Further studies should also include CBCT image registration for further radiation dose reductions.

The image quality assessment is a further limitation of the study. Image quality and vessel contrast depend not only from the angiographic system, but also from the applied flow rate during injection and amount of injected contrast agent for each series. We believe that these incongruences regarding procedure workflow apply to both groups and do not result in a systemic bias. Although the image quality assessment had been adopted from well-established previous studies, it remains a subjective tool. However, the image-assessment results demonstrate a good inter-observer-agreement concerning AlluraClarity $(k=0.77)$ and Allura Xper $(k=0.75)$.

In summary, we have shown that the new angiographic noise reduction imaging technology significantly decreases the radiation dose during adrenal vein sampling without compromising image quality or increasing fluoroscopy time or contrast volume.

\section{References}

1. Stowasser M. Primary aldosteronism in 2011: towards a better understanding of causation and consequences. Nature Reviews Endocrinology 2012; 8:70-72.

2. Rossi GP, Bernini G, Caliumi $C$ et al. A prospective study of the prevalence of primary aldosteronism in 1125 hypertensive patients. J Am Coll Cardiol 2006; 48:2293-2300

3. Douma S, Petidis K, Doumas $M$, et al. Prevalence of primary hyperaldosteronism in resistant hypertension: a retrospective observational study. Lancet. 2008; 371:1921-6.

4. Young WF Jr. Diagnosis and treatment of primary aldosteronism: practical clinical perspectives. J Intern Med.2019;285(2):126-148

5. Daunt M. Adrenal vein sampling: how to make it quick, easy, and successful. Radiographics 2005; 1:S143-58

6. Funder JW, Carey RM, Mantero F, Murad H, Reincke M, Shibata H, Stowasser M, Young Jr, W. The Management of Primary Aldosteronism: Case Detection, Diagnosis, and Treatment: An Endocrine Society Clinical Practice Guideline, The Journal of Clinical Endocrinology \& Metabolism. 2016; Volume 101, Issue 5, Pages 1889-1916.

7. 7. Rossi GP, Auchus R, Brown M, Lenders J, Naruse M, Plouin PF, Satoh F, Young Jr, W. An Expert Consensus Statement on Use of Adrenal Vein Sampling for the Subtyping of Primary Aldosteronism. Hypertension 2014, Volume 63, Issue 1, January 2014, Pages 151-160.

8. Kahn SL, Angle JF. Adrenalveinsampling. Tech Vasc Interv Radiol. 2010;13(2):110-25 
9. Catena C, Colussi G, Di Fabio A et al. Mineralocorticoid antagonist's treatment versus surgery in primary aldosteronism. Horm Metab Res 2010; 42:440-445

10. Satani N, Ota H, Seiji K, et al. Intra-adrenal aldosterone secretion: segmental adrenal venous sampling for localization. Radiology. 2016;278(1):265-74.

11. Fuss CT, Treitl M, Rayes N, Podrabsky P, Fenske WK, Heinrich DA, Reincke M, Petersen TO, Fassnach M, Quinkler M, Kickuth R, Hahner S. Radiation exposureofadrenal vein sampling: a German Multicenter Study. Eur J Endocrinol. 2018 1;179(4):261-267.

12. McAdams HP,Samei E,Dobbins J 3rd, Tourassi GD, Ravin CE. Recent advances in chest radiography. Radiology. 2006;241(3):663-83.

13. Morita S,Endo K, Suzaki S,Ishizaki U, Yamazaki H, Nishina Y, Sakai S. Reduction of Radiation Exposure Using Dynamic Trace Digital Angiography and Spot Fluoroscopy During Adrenal Venous Sampling. Cardiovasc Intervent Radiol. 2017;40(5):697-703

14. Busser WM, Arntz MJ, Jenniskens SF, et al. Image registration of cone-beam computer tomography and preprocedural computertomography aids in localization of adrenal veins and decreasing radiation dose in adrenal vein sampling. Cardiovasc Intervent Radiol. 2015; 38(4):993-7.

15. Chang CC, Lee BC, Chang YC, Wu VC, Huang KH, Liu KL; TAIPAI Study Group. Comparison of C-arm computed tomography and on-site quick cortisol assay foradrenalvenous sampling: A retrospective study of 178 patients. Eur Radiol. 2017;27(12):5006-5014.

16. van Strijen MJ, Grünhagen T, Mauti M, Zähringer M, Gaines PA, Robinson GJ, Railton NJ, van Overhagen $\mathrm{H}$, Habraken J, van Leersum M., Evaluation of a noise reduction imaging technology in iliac digital subtraction angiography: noninferior clinical image quality with lower patient and scatter dose. J Vasc Interv Radiol. 2015; 26(5):642-50.

17. Soderman M, Mauti M, Boon S, Omar A, Marteinsdóttir M, Andersson T, Holmin S, Hoornaert B. Radiation dose in neuroangiography using image noise reduction technology: a population study based on 614 patients. Neuroradiology. 2013; 55(11):1365-72.

18. Dekker LR, van der Voort PH, Simmers TA, Verbeek XA, Bullens RW, Veer MV, Brands PJ, Meijer A. New image processing and noise reduction technology allows reduction of radiation exposure in complex electrophysiologic interventions while maintaining optimal image quality: a randomized clinical trial. Heart Rhythm. 2013; 10(11):1678-82.

19. Schernthaner RE, Duran R, Chapiro J, Wang Z, Geschwind JF, Lin M. A new angiographic imaging platform reduces radiation exposure for patients with liver cancer treated with transarterial chemoembolization. Eur Radiol. 2015; 25(11):3255-62.

20. SpinkC, Avanesov M, Schmidt T, Grass M, Schoen G, Adam G, Koops A, Ittrich H, Bannas P. Noise reduction angiographic imaging technology reduces radiation dose duringbronchial artery embolization. Eur J Radiol. 2017; 97:115-118.

21. SpinkC, Avanesov M, Schmidt T, Grass M, Schoen G, Adam G, Bannas P, Koops A. Radiation dose reduction during transjugular intrahepatic portosystemic shunt implantation using a new imaging technology. Eur J Radiol. 2017; 86:284-288. 
22. Miller DL, Balter S, Noonan PT, Georgia JD. Minimizing radiation-induced skin injury in interventional radiology procedures. Radiology. 2002; 225(2):329-36.

23. Stewart FA, Akleyev AV, Hauer-Jensen M, Hendry JH, Kleiman NJ, Macvittie TJ, Aleman BM, Edgar AB, Mabuchi K, Muirhead CR, Shore RE, Wallace WH. ICRP statement on tissue reactions and early and late effects of radiation in normal tissues and organs-threshold doses for tissue reactions in a radiation protection context. Ann ICRP. 2012; (1-2):1-322.

24. Midgley SM. Capture and analysis of radiation dose reports for radiology. Australas Phys Eng Sci Med. 2014; 37(4):805-19.

25. Beuschlein F. The SPARTACUS Trial: Controversies and Unresolved Issues. Horm Metab Res. 2017 Dec;49(12):936-942

\section{Figures}

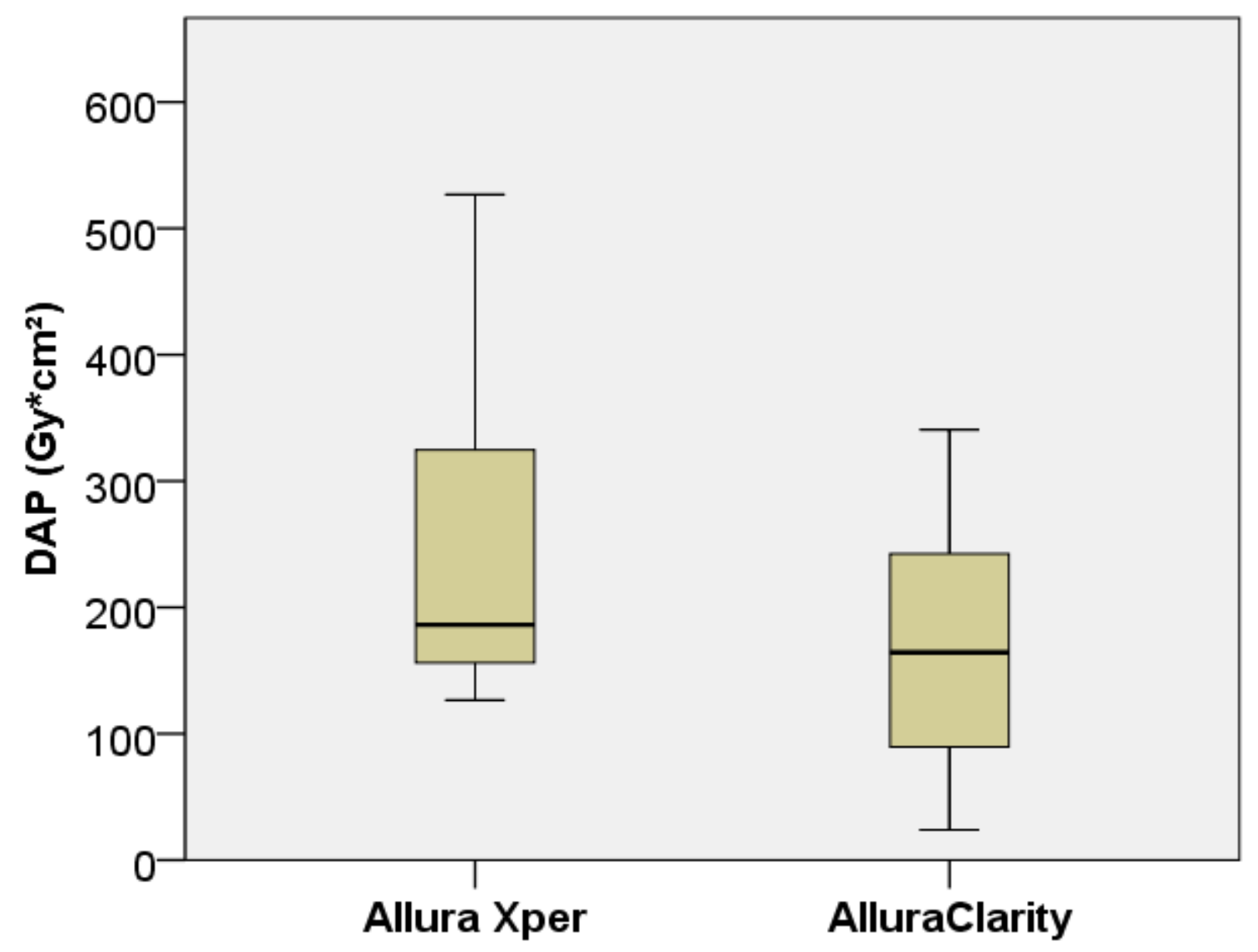

\section{Figure 1}

Box plot showing the mean cumulative dose area product (DAP) during adrenal vein sampling (AVS) for Allura Xper (235.1 $\pm 113.1 \mathrm{Gycm} 2)$ and AlluraClarity $(170.1 \pm 94.5 \mathrm{Gycm} 2)$. DAP includes both digital subtraction angiography and digital fluoroscopy. 


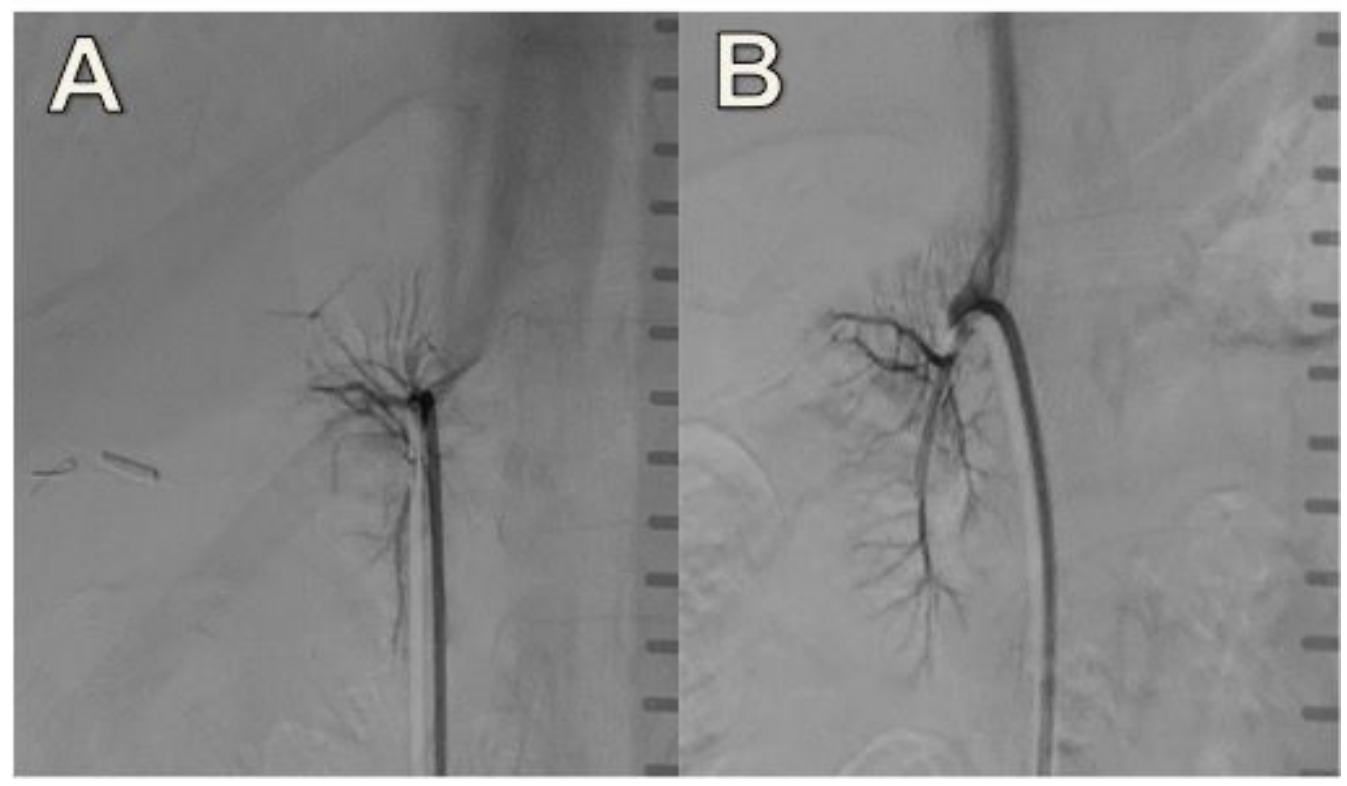

\section{Figure 2}

DSA of selectively catheterized right adrenal veins during AVS using Allura Xper (A) and AlluraClarity (B). A: $54 \mathrm{y}$ female, BMI $27.4 \mathrm{~kg} / \mathrm{m} 2$ using Allura Xper with a cumulative dose area product of $205.2 \mathrm{Gycm} 2$. B: $56 \mathrm{y}$ female, BMI $26.2 \mathrm{~kg} / \mathrm{m} 2$ using AlluraClarity with a cumulative dose area product during of 169.7 Gycm2. Both observers rated the image quality for Allura Xper and for AlluraClarity as $4=e x c e l l e n t$ including a sharp delineation of the adrenal veins with sufficient contrast, contrast stain of the adrenal gland and low level of subjective image noise. 